\title{
Nanobiosensors Based on Localized Surface Plasmon Resonance for Biomarker Detection
}

\author{
Yoochan Hong, ${ }^{1}$ Yong-Min Huh, ${ }^{2,3,4}$ Dae Sung Yoon, ${ }^{1}$ and Jaemoon Yang, 4, 5 \\ ${ }^{1}$ Department of Biomedical Engineering, Yonsei University, Wonju, Gangwondo 220-710, Republic of Korea \\ ${ }^{2}$ Department of Radiology, College of Medicine, Yonsei University, Seoul 120-749, Republic of Korea \\ ${ }^{3}$ Severance Biomedical Science Institute (SBSI), Seoul 120-752, Republic of Korea \\ ${ }^{4}$ YUMS-KRIBB Medical Convergence Center, College of Medicine, Yonsei University, Seoul 120-749, Republic of Korea \\ ${ }^{5}$ Severance Integrative Research Institute for Cerebral \& Cardiovascular Diseases, Yonsei University Health System, \\ Seoul 120-752, Republic of Korea \\ Correspondence should be addressed to Jaemoon Yang, 177hum@yuhs.ac
}

Received 14 March 2012; Revised 5 August 2012; Accepted 5 August 2012

Academic Editor: Miguel A. Correa-Duarte

Copyright (๑) 2012 Yoochan Hong et al. This is an open access article distributed under the Creative Commons Attribution License, which permits unrestricted use, distribution, and reproduction in any medium, provided the original work is properly cited.

Localized surface plasmon resonance (LSPR) is induced by incident light when it interacts with noble metal nanoparticles that have smaller sizes than the wavelength of the incident light. Recently, LSPR-based nanobiosensors were developed as tools for highly sensitive, label-free, and flexible sensing techniques for the detection of biomolecular interactions. In this paper, we describe the basic principles of LSPR-based nanobiosensing techniques and LSPR sensor system for biomolecule sensing. We also discuss the challenges using LSPR nanobiosensors for detection of biomolecules as a biomarker.

\section{Introduction}

To establish optimal therapeutic strategies, both obtaining and analyzing molecular information from diseased patients are key activities in clinical and biomedical studies [1]. In particular, the sensitive detection and quantification of disease-associated biomolecules, such as proteins and nucleic acids, in tissues and biological fluids are crucial for accurate diagnoses and reliable prognoses. At present, abundant diagnostic methods, such as enzyme-linked immunosorbent assays (ELISAs) [2], western blots [3], and polymerase chain reaction (PCR), have been reported [4]. However, these methods have limitations. One major limitation is the need for a labeling process using an antibody, with or without an enzyme or nucleic acid (e.g., a primer for PCR) that is capable of binding to the biomolecule of interest [5]. Moreover, PCR requires an amplification process for the detection of nucleic acid targets, which delays detection [6] and may induce contamination during analysis from the presence of dead bacteria [7]. Thus, various diagnostic biosensors based on nanotechnology have been recently developed for the sensing of low concentrations of biomolecules and the recognition of biomolecular interactions. In general, a nanobiosensor is defined as a device that recognizes biological phenomena at the molecular scale with high affinity and specificity and subsequently transduces signals into quantifiable information [8].

Among the variety of nanobiosensors available, localized surface plasmon resonance-(LSPR-) based nanobiosensors are considered one of the most powerful tools in the biotechnology and biosensor fields. LSPR possesses the specific characteristics of metallic or metalized nanostructured materials, such as precious metal nanoparticles, which can be excited by irradiation with incident photons and is resonant with the collective oscillations of conduction electrons at a specific wavelength. Herein, the peaks of LSPR-related spectra are sensitive to the dielectric medium on the surface of the precious metal nanoparticles that can be used to recognize biomolecules [9]. In particular, nanobiosensors based on LSPR have the following advantages for the detection of biomolecules: (1) high sensitivity via detection of refractive index changes, (2) no labeling requirement because of sensing of spectral shifts, (3) real-time assay accessibility using microfluidic systems, (4) good reproducibility 
using nanoparticle substrates, and (5) low cost and easy instrumental setup. These advantages demonstrate that nanobiosensors can be applied in a wide range of fields, such as medical, food safety, environmental monitoring, and drug screening [10].

In this paper, we will first introduce the optical properties of LSPR in noble metal nanoparticles and provide the physical origins of LSPR and the relationship between the material properties of noble metal nanoparticles and the surrounding dielectric environment. Subsequently, we will describe how LSPR-based sensors are used to detect molecular interactions, including a description of substrate preparation for LSPR sensing (classified into chemical reaction and lithographic methods) as well as both macroscale and nanoscale detection methods for systemic optical instrumental setup. Finally, we will review the applications of LSPR sensors for the detection of disease-related biomolecules as a biomarker.

\section{Fundamentals of LSPR}

To explain the fundamentals of LSPR nanobiosensors, we discuss the basic optical properties of precious metal nanoparticles, which are divided into general plasmons in the bulk state, surface plasmons, and localized surface plasmons. We also describe the physical theories correlated with LSPR signal generation for scattering phenomenon in spherical and nonspherical nanoparticles and for the relationship between refractive index changes and spectral shifts.

2.1. Optical Properties of Noble Metal Nanoparticles. A plasmon is the collective oscillation of free electrons with respect to fixed positive ions in a metal and is one of the most important properties of metals (Figure 1). Reflection occurs when the frequency of light is below the plasma frequency, because the electric field of light is blocked by electrons in the metal. On the other hand, transmittance occurs when the frequency of light is above the plasma frequency, because light cannot be blocked by electrons. In most cases, the plasma frequency of metals is in the ultraviolet region, making the plasma reflective in the visible range. For general plasmons in the bulk state, plasmon energy $E_{p}$ can be represented by

$$
E_{p}=\hbar \sqrt{\frac{n e^{2}}{m \varepsilon_{0}}}=\hbar \cdot \omega_{p},
$$

where $n$ is the electron density, $e$ is the electron charge, $m$ is the electron mass, $\varepsilon_{0}$ is the permittivity of the free space, $\hbar$ is the Planck constant, and $\omega_{p}$ is the plasmon frequency.

Moreover, when the plasmon is refined at the surface of materials, plasmons take the form of surface plasmons. Surface plasmons are confined to the surfaces of metals and interact strongly with light, resulting in polaritons. These occur at the interface of a vacuum or material with a small, positive, imaginary part of the dielectric constant and a large, real part of the dielectric constant. Surface plasmons are optically excited, and light can be coupled into propagating or standing surface plasmons through a grating or defect in the metal surface $[12,14]$. Incident light is most efficiently coupled at a high angle, because it is the oscillating electric field of the incoming plane wave that excites the surface plasmons.

In addition, surface plasmons confined to a nanostructure are called localized surface plasmons (LSPs). LSPs are collective oscillations of electrons in metallic nanoparticles that are excited by incident light. LSPs enhance the electric field near the surface of the nanoparticles; this enhancement is highest at the nanoparticle surface and decays rapidly at the nanoparticle-dielectric background interface. The collective oscillation is maximized at the resonance wavelength, which occurs at the visible wavelength region for precious metal nanoparticles. Enhancements of the electric field and light intensity play important roles in LSPs with respect to high spatial and spectral resolution, limited only by the size of the nanoparticles [21,22].

2.2. Physical Theories Correlated with LSPR. The Mie solution to Maxwell's equations describes the scattering and absorption of incident light by spherical particles [23]. It describes a relationship for the extinction cross-section, $\sigma_{\text {ext }}\left(\sigma_{\text {ext }}=\sigma_{\text {abs }}+\sigma_{\text {sca }}\right)$, that is, the summation of the absorption cross-section and the scattering cross-section of the metal nanoparticles. For small particles $(d \ll \lambda)$, the Mie solution is represented by

$$
\sigma_{\mathrm{ext}}=9 \frac{\omega}{c} \varepsilon_{3 / 2} V_{0} \frac{\varepsilon_{2}(\omega)}{\left[\varepsilon_{1}(\omega)+2 \varepsilon_{m}\right]^{2}+\varepsilon_{2}(\omega)^{2}},
$$

where $V_{0}=(4 \pi / 3) R^{3}, \omega$ is the angular frequency of the extinction radiation, $\varepsilon_{m}$ is the dielectric function of the medium surrounding the metal nanoparticles, and $\varepsilon_{1}$ and $\varepsilon_{2}$ are the real and imaginary parts of the dielectric function of the metal nanoparticles, respectively. As described in (2), the surface plasmon absorption band appears when $\varepsilon_{1}(\omega) \approx$ $-2 \varepsilon_{m}$ if $\varepsilon_{2}(\omega)$ is small or if it is only weakly dependent on $\omega$. The bandwidth and amplitude of the peak are well approximated by $\varepsilon_{2}(\omega)$; however, contrary to experimental cases, size dependency of the position is not predicted by (2). The size dependency of the position of the surface plasmon absorption band of the metal nanoparticles can be adapted by assuming size-dependent dielectric functions [24].

For a more sensitive detection of the LSPR signal, nanoparticle shape is an important factor. There are many kinds of nanoparticles: nanospheres, nanodiscs, nanopyramids, and nanorods. Among these variations, nanorods offer a higher sensitivity to refractive index changes than nanospheres. The longitudinal LSPR signal for a nanorod with an aspect ratio of 3 was shown to have a 6 -fold higher sensitivity [25]. For this reason, we further describe the physical theory for nonspherical nanoparticles. In cases of nonspherical nanoparticles, the extension of the Mie theory, that is, the Gans theory, is applicable (Figure 2). This theory provides the scattering characteristics for both oblate and 


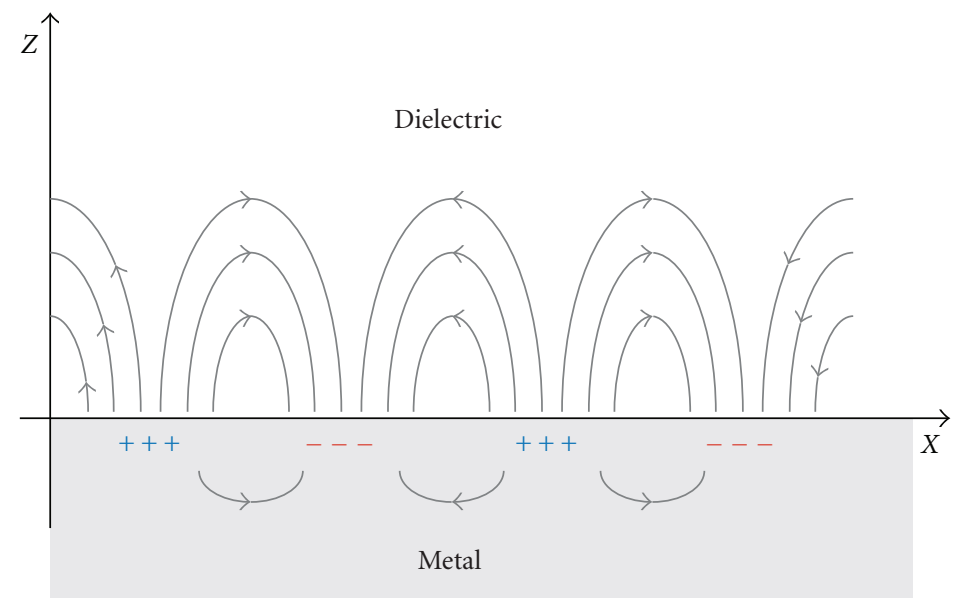

(a)

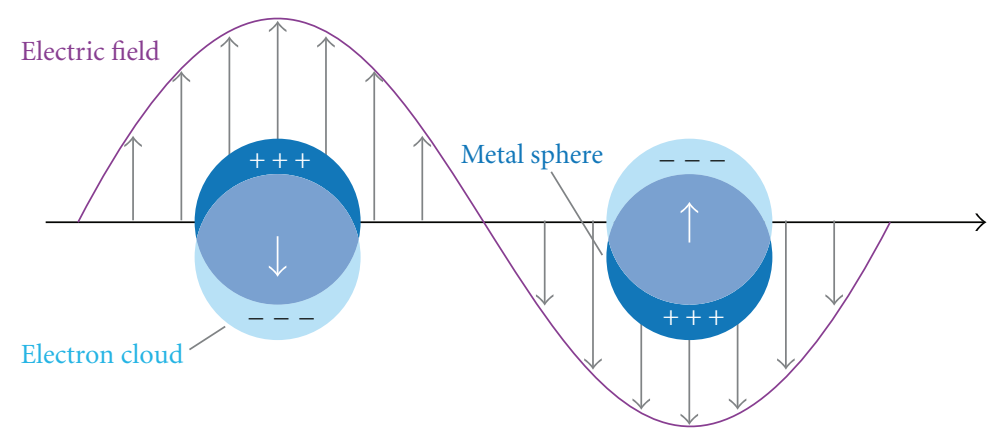

(b)

FIGURE 1: Illustrations of (a) surface plasmon resonance (SPR) and (b) localized surface plasmon resonance (LSPR), resulting from the collective oscillations of delocalized electrons in response to an external electric field. Reprinted with permission from [14]. Copyright 2007 Annual Review of Physical Chemistry.

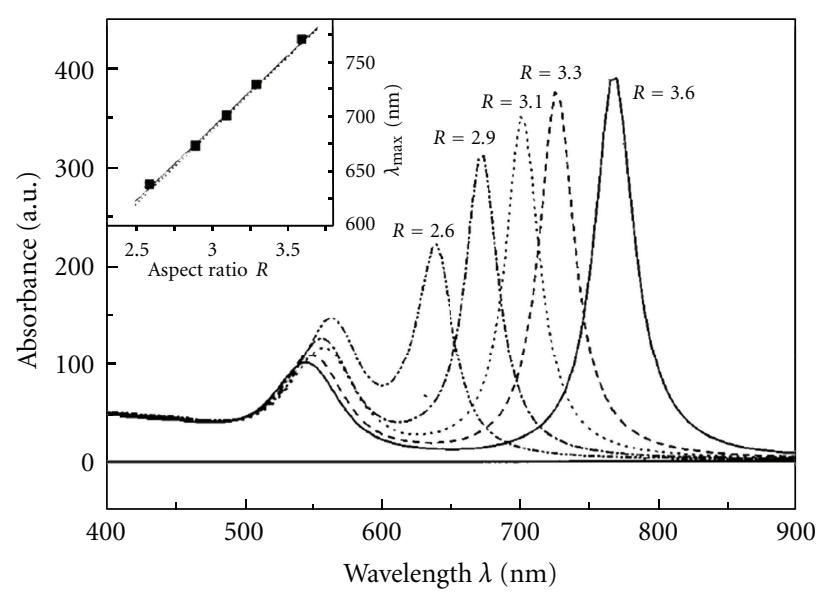

(a)

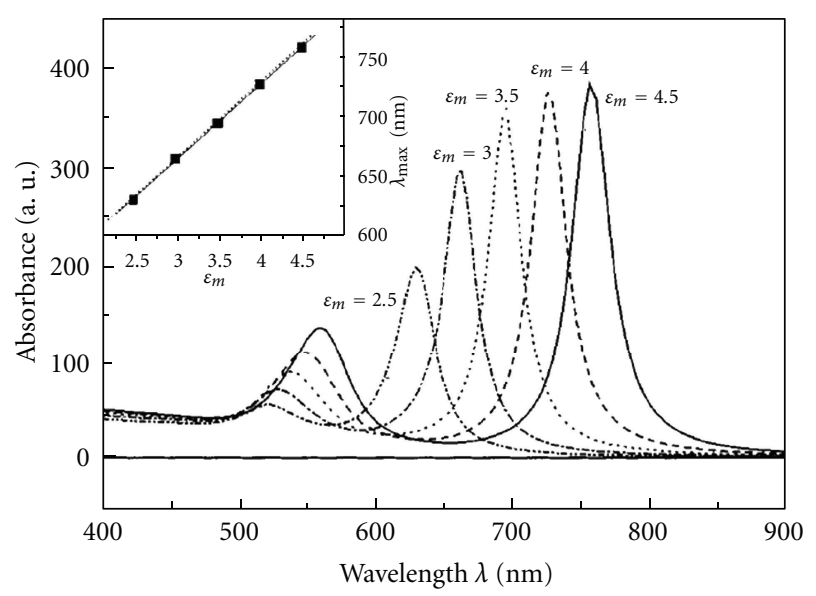

(b)

FIGURE 2: The calculation of Gans theory for gold nanorods. (a) Dependence of the absorbance spectrum on aspect ratio for a constant dielectric constant of the medium (inset: peak wavelength increases linearly with aspect ratio). (b) Dependence of the absorbance spectrum on the dielectric constant of the medium for a constant aspect ratio (inset: peak wavelength increases linearly with dielectric constant). Reprinted with permission from [11]. Copyright 1999 American Chemical Society. 


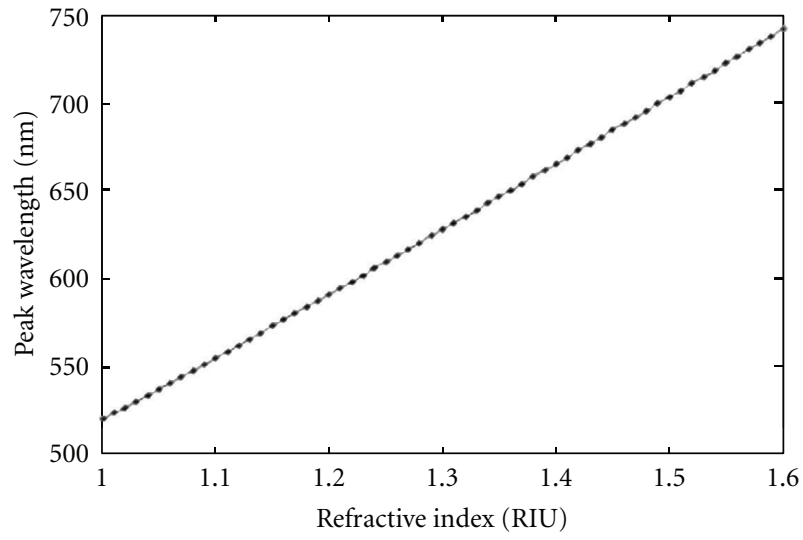

Figure 3: The Drude model calculation. The graph shows the linear dependence of LSPR wavelength on the refractive index of the surrounding medium. Reprinted with permission from [12]. Copyright 2011 American Chemical Society.

prolate spheroidal nanoparticles [26, 27], describing the absorption cross-section for a prolate spheroid as

$$
\sigma_{\mathrm{abs}}=\frac{\omega}{3 c} \varepsilon_{m}^{3 / 2} V \sum_{j} \frac{\left(1 / P_{j}^{2}\right) \varepsilon_{2}}{\left[\varepsilon_{1}+\left\{\left(1-P_{j}\right) / P_{j}\right\} \varepsilon_{m}\right]^{2}+\varepsilon_{2}^{2}},
$$

where $j$ represents the three dimensions of the particle and $P_{j}$ includes $P_{A}, P_{B}$, and $P_{C}$, termed depolarization factors, for each axis of the prolate spheroid particle. The depolarization factors anisotropically alter the values of $\varepsilon_{1}$ and $\varepsilon_{2}$, and the resulting LSPR peak frequencies are represented as

$$
\begin{gathered}
P_{A}=\frac{1-e^{2}}{e^{2}}\left[\frac{1}{2 e} \ln \left(\frac{1+e}{1-e}\right)-1\right], \\
P_{B}=P_{c}=\frac{1-P_{A}}{2},
\end{gathered}
$$

where $e$ is the following factor, including the aspect ratio $R$ of the particle $[11,28,29]$

$$
e=\left[1-\left(\frac{B}{A}\right)^{2}\right]^{1 / 2}=\left(1-\frac{1}{R^{2}}\right)^{1 / 2} .
$$

The extinction spectrum resulting from (3) has two peaks, one corresponding to the transverse plasmon peak from the contributions of the $x$ - and $y$-axes to the sum, and the other corresponding to the longitudinal plasmon peak from the $z$-axis contribution. Equation (3) also provides an intuitive understanding of the effects of aspect ratio on LSPR peak wavelength. Factor $\varepsilon_{m}$, which is 2 for spherical particles, is $\left[\left(1-P_{j}\right) / P_{j}\right]$, a quantity that increases with aspect ratio and can be much greater than 2 . This leads to a red shift of the plasmon peak with increasing aspect ratio, as well as increased sensitivity to the dielectric constant of the surrounding medium.

As mentioned above, LSPR-based sensors fundamentally detect spectral shifts by changes in the localized refractive index in surrounding dielectric environments (Figure 3).
The wavelength of the LSPR peak is dependent on the dielectric function of the surrounding medium [30]. This phenomenon can be proven using the Drude model, represented as

$$
\varepsilon_{1}=1-\frac{\omega_{p}^{2}}{\omega^{2}+\gamma^{2}},
$$

where $\omega_{p}$ is the plasmon frequency and $\gamma$ is the damping factor of the bulk metal. In the visible and near-infrared regions, in the case of $\gamma \ll \omega_{p}$, (6) is simplified to

$$
\varepsilon_{1}=1-\frac{\omega_{p}^{2}}{\omega^{2}}
$$

Under resonance conditions $\left(\varepsilon_{1}=-2 \varepsilon_{m}\right)$, then

$$
\omega_{\max }=\frac{\omega_{p}}{\sqrt{2 \varepsilon_{m}+1}},
$$

where $\omega_{\max }$ is the frequency of the LSPR peak. Substituting frequency with wavelength via $\lambda=2 \pi c / \omega$, and then dielectric constant with refractive index via $\varepsilon_{m}=n^{2}$, (8) becomes

$$
\lambda_{\max }=\lambda_{p} \sqrt{2 n_{m}^{2}+1},
$$

where $\lambda_{\max }$ is the wavelength at the LSPR peak and $\lambda_{p}$ is the wavelength corresponding to the plasma frequency of the bulk metal. Thus, we find an approximately linear relationship between the wavelength of the LSPR peak and refractive index; this relationship can be applied to highsensitivity detection at the molecular level and, furthermore, to quantifiable bioassays using LSPR-based sensors.

\section{Hardware Setup of LSPR}

The application of high-sensitivity LSPR in surrounding dielectric environments has advantages for detection at the molecular level. In this section, we discuss the components of LSPR-based nanobiosensor systems for high-sensitivity recognition of biomolecules. First, we describe the dependency of nanoparticles on various conditions, such as size and shape, for detection of high-sensitivity LSPR signals. Then, we discuss substrate preparation methods, that is, adsorption and lithographic techniques, and close with LSPR signal detection methods and ways to improve their limits of detection.

3.1. Preparation of Nanostructures. The spectral properties of LSPR signals are contributed by the shape and size of the nanoparticles due to surface polarization [13] (Figure 4). Many reports have described the use of various nanoparticle shapes as well as nanoparticle arrays coated with thin films. The size of the nanoparticles influences the relative magnitude of both absorption and scattering cross-sections. Moreover, the increase in edges or sharpness of a nanoparticle results in a red shift of extinction spectra due to an increase in charge separation, whereas increased symmetry results in increases in LSPR signal intensity [31]. The number of modes in which a given nanoparticle can be polarized 


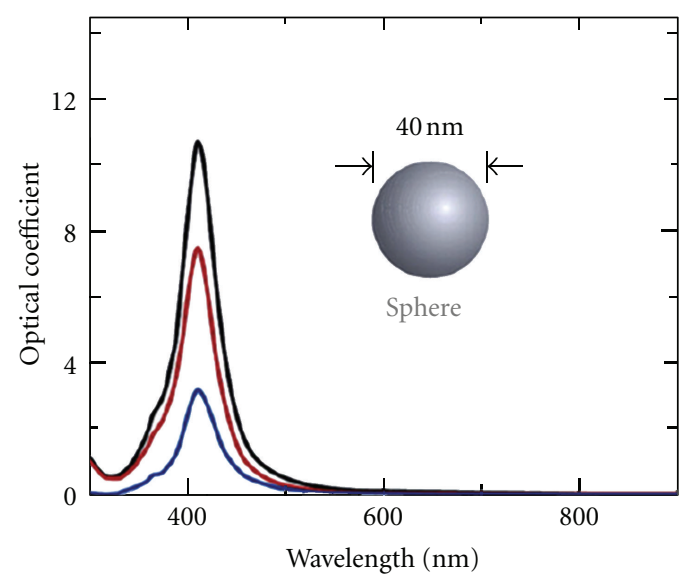

(a)

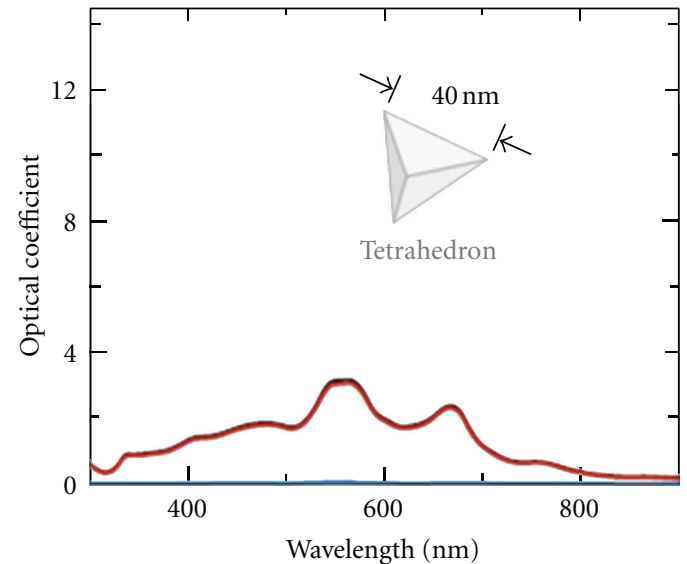

(c)

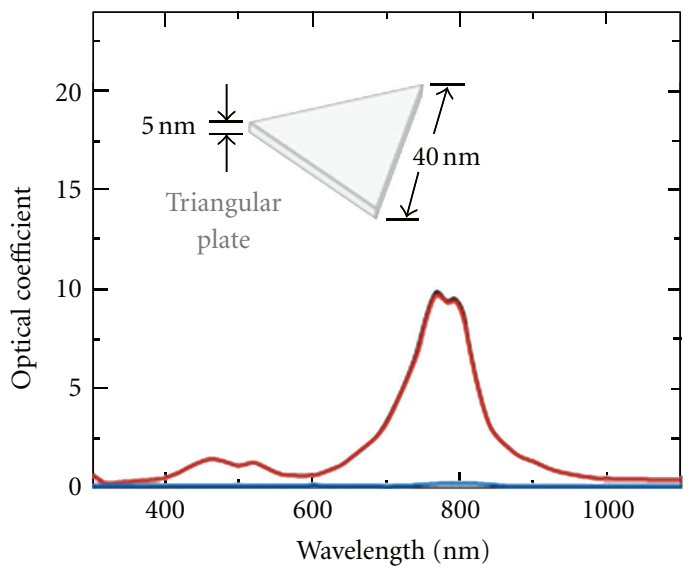

(e)

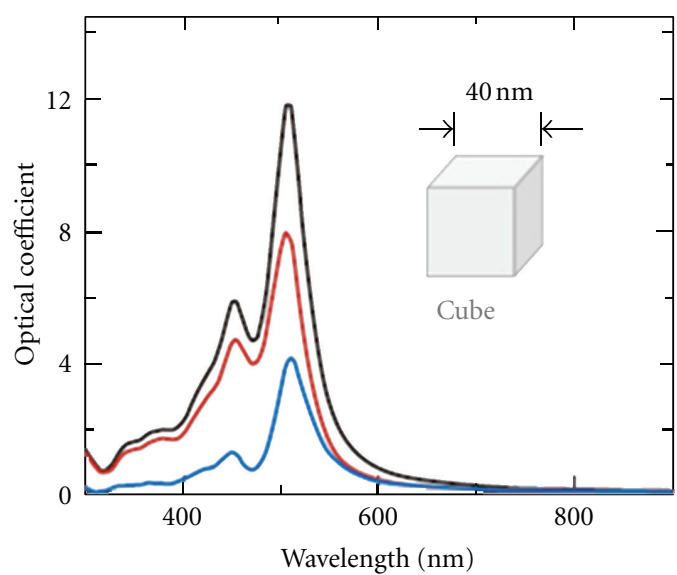

(b)

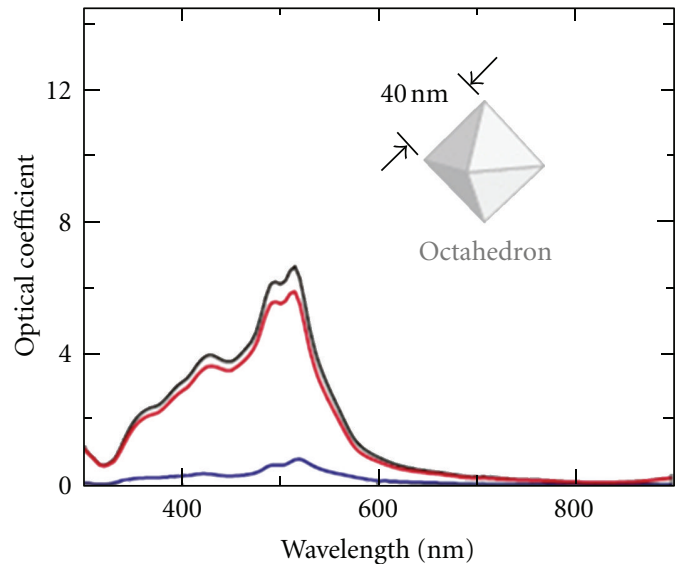

(d)

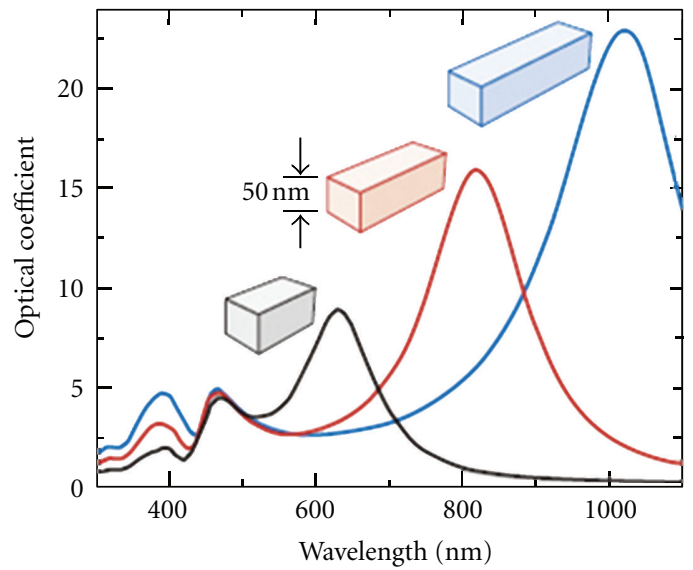

(f)

Figure 4: Extinction (black), absorption (red), and scattering (blue) spectra calculated for Ag nanoparticles of different shapes: (a) sphere, (b) cube, (c) tetrahedron, (d) octahedron, and (e) triangular plate. (f) Extinction spectra of rectangular bars with aspect ratios of 2 (black), 3 (red), and 4 (blue). Reprinted with permission from [13]. Copyright 2011 Elsevier.

determines the resonance absorption peaks [25, 31, 32]. Thus, nonspherical nanoparticles tend to exhibit multiple red-shifted peaks compared to nanospheres.

As previously mentioned, refractive index sensitivity for spheroidal nanoparticles is determined not only by material composition and particle size but also by aspect ratio. Deviations in aspect ratio are more influential on the spectral shift of the LSPR signal than are changes in size. For example, changing the size from 10 to $100 \mathrm{~nm}$ for nanospheres results in a red shift of $47 \mathrm{~nm}$; in contrast, changing the aspect ratio 
from 2.5 to 3.5 for elliptical nanoparticles results in a red shift of $92 \mathrm{~nm}$ for a longitudinal peak [32]. This example shows that changes in aspect ratio correlate with much smaller changes in the overall size of the nanoparticles. In addition, refractive index sensitivity of LSPR-based sensors also depends on the shape of the nanoparticles, which are highest for nanorods, followed by triangles, and then spheres [33].

\subsection{Fabrication of Substrate for LSPR Signal Sensing.} Nanofabrication techniques are classified into bottom-up and top-down methods, where the former is based on chemical reactions giving rise to nanomaterials with compositions, sizes, and shapes determined by the reaction conditions, and the latter involves the use of lithographic techniques, that is, the synthesis of gold and silver nanostructures (Figure 5).

In the case of metal nanoparticles, the most common method involves the chemical reduction of metallic salts in the presence of stabilizers and surfactants, which allows control of particle orientation, particle shape, and colloidal stability. There are numerous methods that reproducibly control shape and morphology of nanoparticles, allowing a fine tuning of LSPR wavelength [34]. The required bioconjugation of such nanoparticles is a complex process due to the delicate balance between attractive and repulsive electrostatic forces at interfaces between nanoparticles and solutions. Changes in environmental conditions, such as temperature, ionic strength, and $\mathrm{pH}$, can lead to nanoparticle aggregation. To avoid these difficulties, one of the LSPR substrate preparation methods is adsorption of nanoparticles onto the substrate. Nanoparticle adsorption onto substrates is conducted by chemical binding, typically present on glass surfaces, using the high affinity of gold or silver nanoparticles toward specific functional groups, such as amino or mercapto groups [35-37].

Although the nanoparticle adsorption method has been widely used, this method has limitations with respect to polydispersity and reproducibility. To overcome these limitations, lithographic techniques were introduced. Many lithographic techniques have been used to achieve uniformity in size and shape of metal nanostructures. Among them, one technology is electron beam lithography. This technique allows the preparation of substrate for precise control of size, shape, and spatial distribution, generating greater than $99 \%$ monodispersive populations of nanoparticles, owing to its high resolution $[38,39]$. Briefly, a glass slide, supporting a conductive film such as indium tin oxide, is coated with an electron-sensitive photoresist, such as poly(methyl methacrylate) (PMMA). The electron beam is then used to burn off the polymer in a desired pattern, and the exposed surface is chemically developed. Gold or silver is deposited by thermal evaporation, and PMMA is removed by acetone, leaving behind the desired pattern [24]. This technique, as well as focused ion beam lithography, is time consuming and produces only small patterned regions [39]. An alternative, cost-effective technique is nanosphere lithography (NSL), which produces 2D periodic arrays and metal films-over-nanosphere (FON) structures. Due to their substantial roughness, FON structures are extremely efficient surface enhanced Raman scattering substrates. The NSL process is based on the self-assembly of polymer nanospheres arranged in a closely packed hexagonal pattern that is subsequently overcoated with metal [40]. Metal deposited over the mask with a thickness between 15 and $100 \mathrm{~nm}$ generates honeycomb lattices of triangular islands once the mask is washed away. The dimensions of these islands can be controlled by the gap size between spheres and are dependent on the initial bead size [41]. Nanosphere masks formed by polystyrene nanospheres upon solvent evaporation are prone to defects due to size dispersion, point defects (missing nanospheres), line defects (nanosphere slips), and random arrangement. Angle-resolved NSL, also known as shadow NSL, uses annealed polystyrene spheres to expand the range of accessible nanoparticle morphologies to include nanodisks, nanorings, nanoholes, and cup-like structures. Other nanofabrication techniques include laser ablation, based on photo- and radiolytic reduction of metal ions, electrodeposition, and variations of lithographic techniques (e.g., electrostatic colloidal and soft interference). An interesting example of an electrochemical deposition technique was reported by Bok et al. [42] for preparation of $\mathrm{Au} / \mathrm{Ag}$ alloy nanorods with multiple LSPR modes. Tunability of the resonance wavelength was achieved by varying the proportional composition of two noble metals in the plating solution.

3.3. Instrumental Setup for LSPR Sensors. Typically, LSPR instrumental hardware setups are custom-built systems that are classified as macroscale or nanoscale detection methods, depending on the number of observed particles (Figure 6).

Macroscale detection methods are composed of three major parts: a light source, a sample chip, and a spectrometer. The light source, generally a white light source, is passed toward a transparent sample chip that houses a large number of nanoparticles. The passed light source, which contains information about the extent of analyte adsorption, then arrives at its destination, the spectrometer. For nontransparent substrates, such as enclosed FON substrates, reflectionbased optical systems are suitable to study LSPR signals using a macroscale detection method.

On the other hand, nanoscale detection methods are achieved using more delicate and sophisticated systems, such as dark field microscopy-based optical systems. Incident white light hits the surface of the sample at a high angle, and scattered light is collected at a low angle. These two angles, that is, two apertures with different optical geometries, rely on a dark field condenser with a high numerical aperture (NA) that brings incident light obliquely to the surface of the sample; an objective lens selected for suitable NA is used to collect the scattered light. A dark field objective can also be used in reflection mode in which incident and collected light travel through the same objective. The scattered light is then sent to the spectrograph and imaging detector; the most common imaging detector is a camera that uses a CCD chip [13]. Nanoscale detection methods have some advantages: (1) low sample volume, (2) simultaneous 


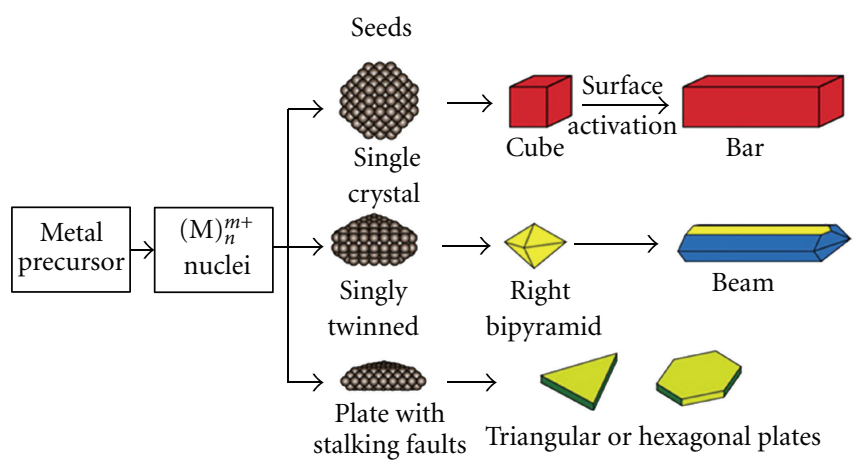

(a)

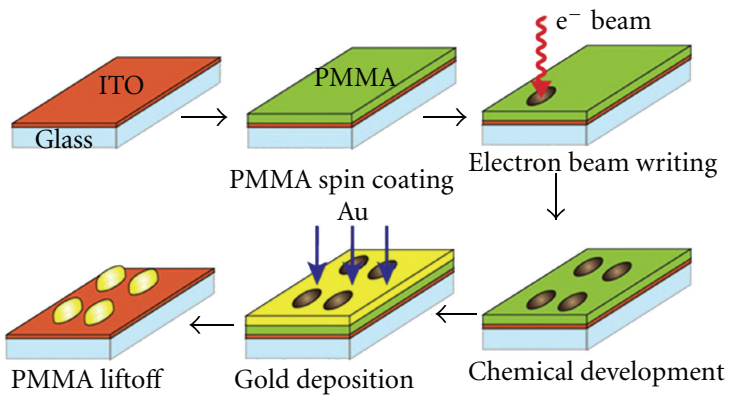

(b)

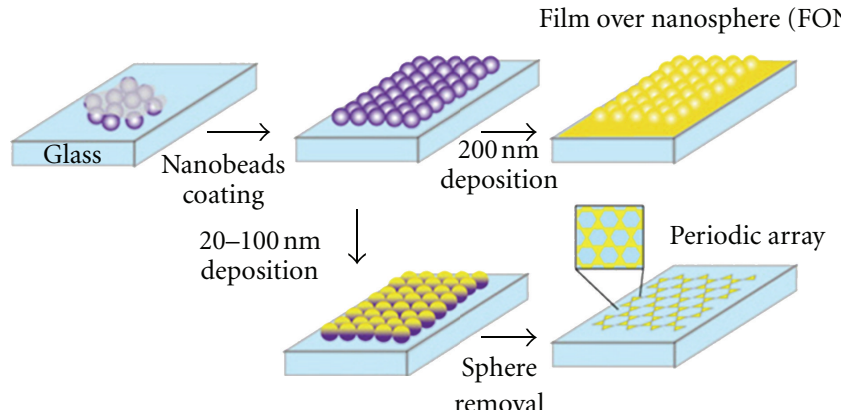

(c)

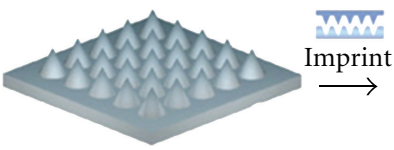

Silicon cones

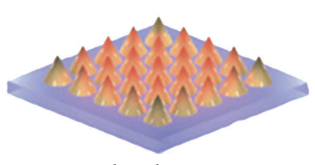

Au coated polymer cones

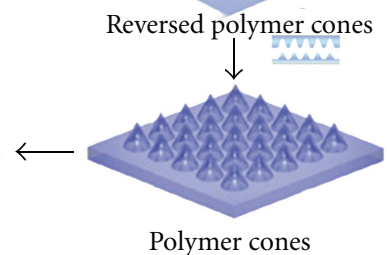

(d)

FIGURE 5: Various substrate fabrication techniques. (a) Wet seed-mediated chemical reduction method. (b) Principle of electron beam technique. (c) Nanosphere lithography process. (d) Schematic of the nanofabrication process for 3D gold-coated nanocones by nanoimprint lithography. Reprinted with permission from [13]. Copyright 2011 Elsevier.

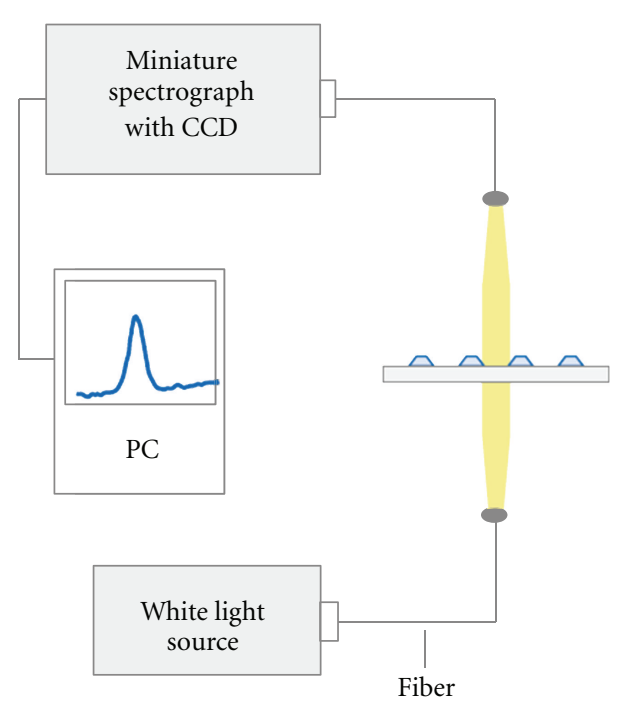

(a)

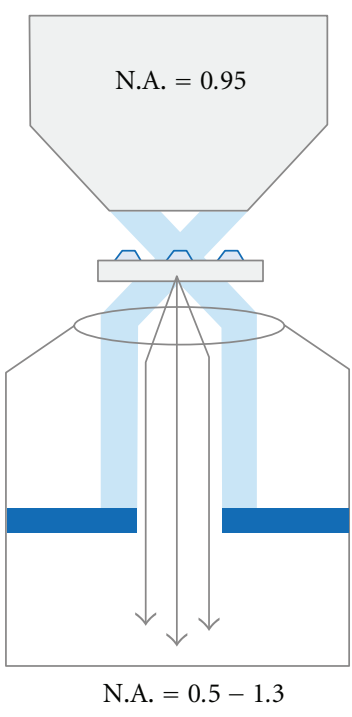

(b)

FIGURE 6: (a) Macroscale setup for measuring the extinction spectrum of nanoparticle arrays. (b) Nanoscale setup for measuring the scattering spectrum of a single nanoparticle. Reprinted with permission from [14]. Copyright 2007 Annual Review of Physical Chemistry. 
detection of multiplexed nanoparticles that have specific wavelength peaks, (3) tracking of each nanoparticle in real time, which allows the trajectory to be determined in biologically amenable solutions, and (4) nanoparticle imaging without photobleaching.

A report by Dahlin et al. [43] describes improvements in the resolution of LSPR instruments. The setup in this study allowed the detection of LSPR shifts with a noise level less than $5 \times 10^{-4} \mathrm{~nm}$ and less than $5 \times 10^{-6}$ extinction units for 2 -second acquisitions. Using nanohole arrays functionalized with biotin, these authors measured neutravidin binding kinetics in real time with a detection limit of less than $0.1 \mathrm{ng} / \mathrm{cm}^{2}$. This level of precision, compared with SPR sensors, provides a signal-to-noise ratio of approximately 2,000 for typical protein-binding reactions.

This improvement in resolution resulted from the collection of more photons in incident light using photodiode arrays with high saturation levels to reduce shot noise as well as from the use of improved fitting algorithms to calculate $\lambda_{\max }$ in real time. By collecting greater numbers of photons, it was possible to determine LSPR peak positions with much greater accuracy than with either the grating resolution of the spectrometer (a few nanometers for commonly used fibercoupled portable spectrometers, depending on the slit width and gratings) or full-width LSPR spectra at half-maximal values. The use of photodiode array spectrometers with highsaturation capacities has, therefore, greatly enhanced LSPR resolution. This high resolution enables the detection of weaker signals, improving the measurements of real-time binding kinetics.

\section{Sensing of Biomarkers Based on LSPR}

Recently, various researches for biological assays using LSPR sensors have been reported to detect biomolecules and enhance the sensing efficiency. In particular, the quantification and analysis of biomolecules as a biomarker have demonstrated great promise for accurate diagnoses and highly reliable prognoses $[15,16,44,45]$. Furthermore, the recognition of biomolecules can be used to understand fundamental biochemical and biomedical processes involved in diseases and monitor patient responses after the treatment [46]. The traditional immunoassay methods, such as ELISA [2] and fluorescence immunoassays [47, 48], permit reliable biomedical diagnostics, but the increased demand for early and precise screening methods of molecular biomarkers is pushing the development of ultrasensitive sensors through signal amplification or exploitation of novel detection techniques [49]. LSPR sensors are one of the most powerful technologies for ultrasensitive, real-time, and multiplex sensing. Here, we thus discuss the applications of LSPR-based nanobiosensors for the detection of molecular biomarkers.

To confirm the capability about sensitive detection of molecular biomarker using LSPR sensor, biotin-streptavidin interaction is commonly used as a model system [50-52]. The interaction between biotin and streptavidin forms a strong, specific bond, and many reagents for this bioconjugation technique are readily available [53-56]. The biotinstreptavidin interaction is notably well suited for LSPR sensors, because biotin is a relatively small organic molecule and can be conjugated to the nanoparticle surface [57]. In contrast to biotin, streptavidin is a relatively large protein and is easily detected by measuring changes in its refractive index $[58,59]$. There have been many investigations of biotinstreptavidin interactions using LSPR sensors [60-64]. In one of these examples [51], biotin was attached through a linker to gold nanospheres that were previously immobilized onto a glass substrate. Detection was performed by monitoring spectral changes using a spectrometer or by detecting a color intensity changes using a commercial flatbed scanner. Recently, Chen et al. [61] introduced single-particle LSPR imaging utilizing transmission grating to generate diffraction spectra from multiple nanoparticles captured using a CCD camera. This system detected biotin-streptavidin interactions with $150 \mathrm{nM}$ streptavidin concentrations and a spectral shift of $13.5 \mathrm{~nm}$. Kaur and Forrest [63] reported that the biotin-streptavidin interaction is dependent on the size of nanoparticles compared with IgG and protein A. Their results indicated that nanoparticle size strongly influences the apparent thickness and refractive index of the adsorbed proteins. They suggested that differences in layer thickness simply correlated to the orientation of the adsorbed proteins and their resulting areal densities.

The utility of LSPR sensors has been demonstrated in an immunoassay format, such as antigen-antibody interactions $[15,16]$. Moreover, antigen-antibody interactions are appropriate to be used in LSPR sensors for real-time analyses [65-67]. Due to its strong binding affinity, the binding kinetics of the biotin-streptavidin interaction are limited by diffusion, and the unbinding kinetics are terribly slow [68-70]. However, antigen-antibody kinetics possess more reasonable binding rates, so LSPR sensors have been used to conduct analyses of binding kinetics in real time [71-73]. Furthermore, real-time analyses have been used to demonstrate specificity within one chip containing the same nanoparticles [74-77]. Based on these techniques, recently, LSPR sensing technology has been applied to diagnose the specific disease [78-82].

Mayer et al. [70] conducted experiments using LSPR sensors that were exposed to a nonspecific and subsequently to a specific antigen, both of which had the same concentrations and molecular weights. These experiments provided a more direct confirmation that antigen-antibody interactions occur on the sensor than did dose-response studies. Haes et al. [15] developed an LSPR biosensor capable of biomarker detection for Alzheimer's disease, that is, detection of amyloid-derived diffusible ligands (ADDLs), at picomolar concentrations (Figure 7(a)). ADDL-anti-ADDL antibody binding was observed in real time, and the presence of the ADDL building block, $\mathrm{A} \beta$, was confirmed using integrated mass spectroscopy spectra. The results of these spectra showed that $\mathrm{A} \beta$ was present in both oxidized and reduced forms, which were hypothesized to be important in Alzheimer's disease progression. Chen et al. [16] utilized a combination of LSPR refractive index sensing and ELISA 


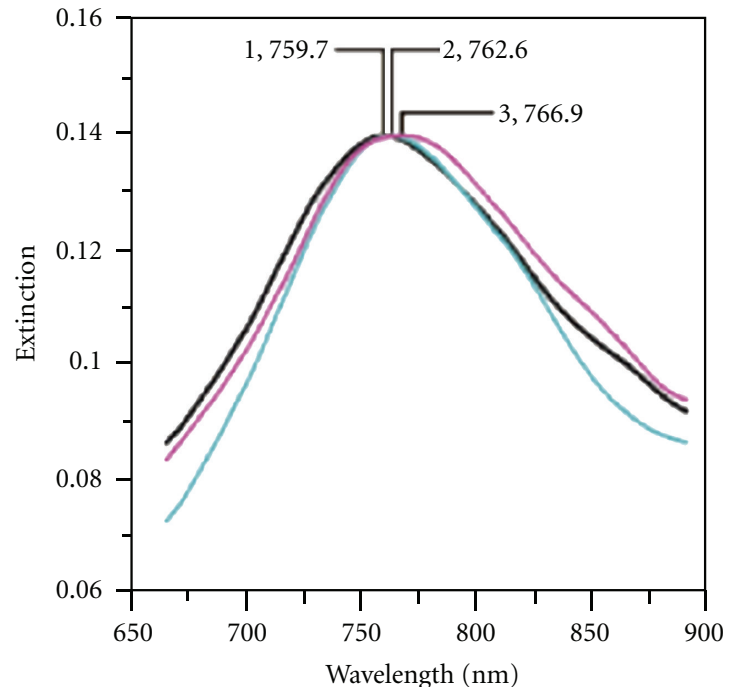

(a)

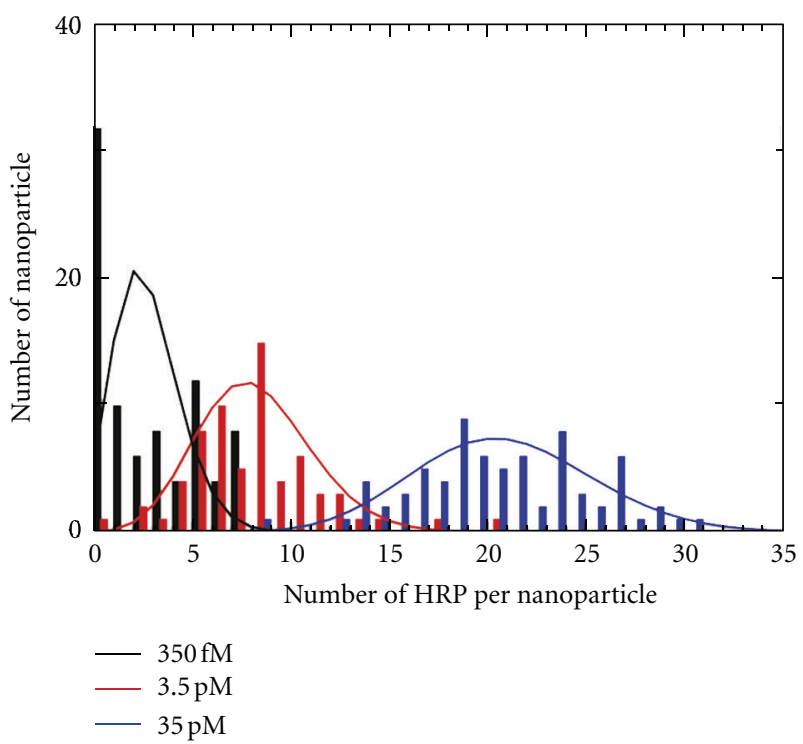

(c)

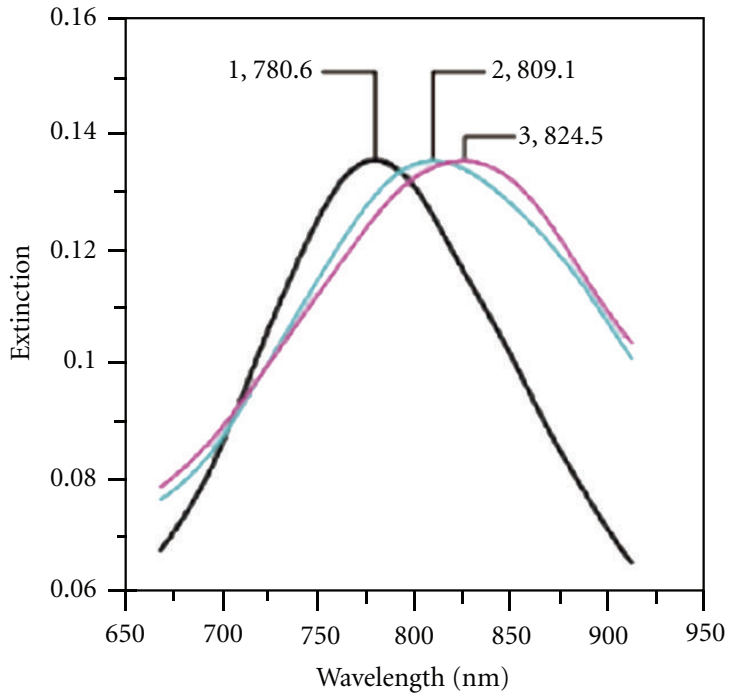

(b)

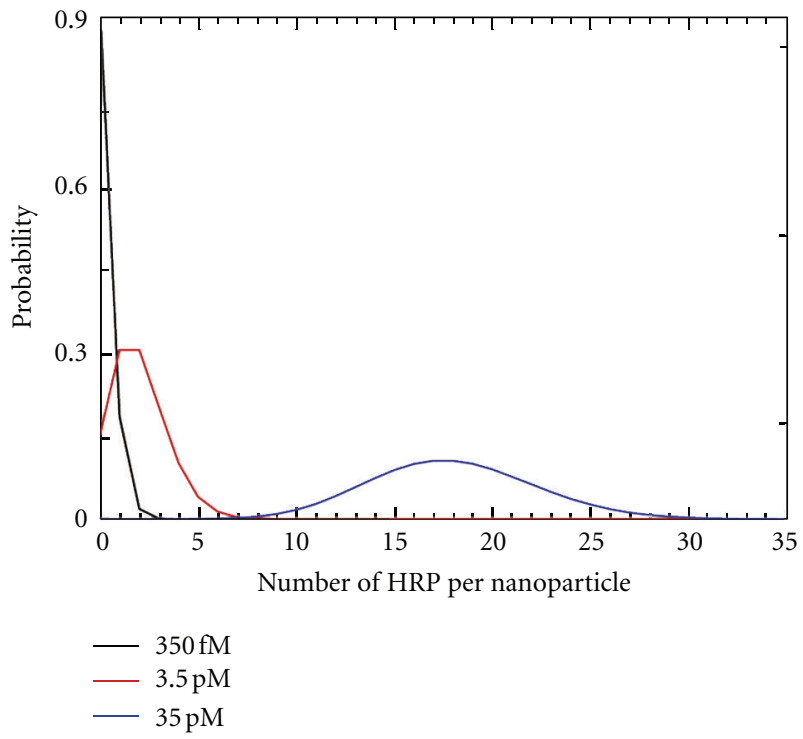

(d)

FIgURE 7: (a) Aging patient: LSPR spectra for each step of the assay. Ag nanoparticles after functionalization with (a-1) anti-ADDL, (a-2) CSF, and (a-3) anti-ADDL. (b) Alzheimer's disease patient: LSPR spectra for each step of the assay. Ag nanoparticles after functionalization with (b-1) anti-ADDL, (b-2) CSF, and (b-3) anti-ADDL. Reprinted with permission from [15]. Copyright 2005 American Chemical Society. (c) Number of HRP molecules per particle estimated. (d) Poisson distributions generated from simulations of HRP diffusion to a single nanoparticle. Reprinted with permission from [16]. Copyright 2011 American Chemical Society.

to develop a simple colorimetric biosensing methodology with single-molecule sensitivity. The technique is based on spectral imaging of a large number of isolated gold nanoparticles. Each particle binds a variable number of horseradish peroxidase (HRP) enzyme molecules that catalyze a localized precipitation reaction at the particle surface. The enzymatic reaction dramatically amplifies the shift of the LSPR scattering maximum, $\lambda_{\max }$, and makes it possible to detect the presence of only one or a few HRP molecules per particle (Figure 7(b)).

Specifically, LSPR-based biomarker sensing techniques have been used in cancer diagnosis in recent years because the sensing of molecular biomarkers has shown promise in the early detection of disease, recognition of malignant conditions, and monitoring of the therapeutic efficacy (Table 1) [15, 16, 44]. Huang et al. [17] introduced singlemolecule nanoparticle optical biosensors, which consist of controlled, single-monoclonal antibody molecules attached to individual nanoparticles. Such tiny nanosensors offer detection of single molecules present in a volume of individual nanoparticles that are smaller than the crosssectional area of the antibody molecules. These authors also found that the biosensors resisted photodegradation and could be used for imaging and quantitative analyses 
TABLE 1: Applications for sensing of cancer-related biomolecules using LSPR sensors.

\begin{tabular}{|c|c|c|c|c|}
\hline Biomolecules & LSPR particles & Spectral shift & Detection limit & References \\
\hline Tumor necrosis factor & Ag nanosphere & $29 \mathrm{~nm}$ & $200 \mathrm{ng} / \mathrm{mL}$ & [17] \\
\hline Prostate-specific antigen, (PSA) & Au nanodisc & $2.2 \mathrm{~nm}$ & $10^{-8} \mathrm{M}$ & {$[18]$} \\
\hline p53 (from head and neck squamous carcinoma) & Triangular Ag nanoparticle & $88 \mathrm{~nm}$ & $59.45 \mathrm{pg} / \mathrm{mL}$ & {$[19]$} \\
\hline PSA & Au nanosphere & $2.75 \mathrm{~nm}$ & $0.1 \mathrm{pg} / \mathrm{mL}$ & {$[20]$} \\
\hline
\end{tabular}

of single-protein molecules, tumor necrosis factor- $\alpha$, and binding reactions for hours in real time. Chen et al. [18] demonstrated an LSPR-based biomolecular detection system that is simple and cost effective and, at the same time, is characterized by ultrahigh sensitivity (i.e., to several tens of attomoles of the analyte detected per $\mathrm{cm}^{2}$ of the sensor surface). The chosen clinical target analytes for detection were extracellular adherence protein, found on the outer surface of Staphylococcus aureus, and prostatespecific antigen (PSA). Zhou et al. [19] applied a developed LSPR biosensor based on triangular silver nanoparticles for the detection of serum levels of the nuclear tumor suppressor protein, p53, in samples from head and neck squamous cell carcinoma patients. This report showed that LSPR nanobiosensors provide a promising platform with attractive advantages for serological or molecular diagnosis of the tumor. Hwang and Sim [20] described a strategy for the sensitive detection of target protein biomarkers based on LSPR responses to a single gold nanosphere in combination with signal enhancement by polyclonal antibodies. Changes in the dielectric constant of the surrounding microenvironment induced by the immune reaction between the target antigen and the polyclonal antibody created an additional spectral shift. A complex of PSA- $\alpha_{1}$-antichymotrysin was chosen as a target analyte, given the important role of PSA in prostate cancer diagnosis. The LSPR responses of the single $\mathrm{Au}$ nanoparticles were monitored after the introduction of that complex at various concentrations $(0.01 \mathrm{pg} / \mathrm{mL}-1 \mathrm{ng} / \mathrm{mL})$. Those examples provide insight into molecular biomarker detection using LSPR nanobiosensors with high sensitivity via detection of refractive index changes in localized metal surfaces, nonlabeling methods via sensing of the spectral shift of the maximum peak, and accessibility of real-time, multiplexed assays using microfluidic systems and nanoparticle arrays.

\section{Conclusion}

This paper has highlighted the physical theories and applications of LSPR nanobiosensors. By controlling and tuning the optical behaviors described in the aforementioned physical theories, such as the material, size, shape, and composition of noble metal nanoparticles, the sensitivity of LSPR nanobiosensors may be improved. Various approaches with regard to substrate preparation and optical instrumental setup have been presented to illustrate the challenges and suggestions for improvement in detection sensitivity for LSPR sensors. Narrower bandwidth and higher spectral sensitivity are commonly accomplished by lithographic methods. However, these methods are relatively expensive for manufacturing and small-size applications. By measuring of LSPR signals using macroscale detection methods, an average signal induced by numerous nanoparticles can be obtained. Nanoscale detection methods need only a few particles to be monitored simultaneously, so the required sample volume is significantly lower for macroscale detection methods. The various examples of biomolecular detection with highresolution sensitivity using LSPR have been reported; thus, LSPR-based nanobiosensors show promise as powerful tools for challenging problems in clinical and biomedical applications.

\section{Acknowledgment}

This research was supported by the National Research Foundation of Korea (NRF) funded by the Ministry of Education, Science and Technology (2012R1A1A2006248, 2010-0019923), a grant of the Korea Healthcare technology R\&D Project, Ministry for Health\&Welfare Affairs, Republic of Korea (A085136), and a faculty research grant of Yonsei University College of Medicine for 2012. (8-2012-0013).

\section{References}

[1] L. Nicu and T. Leichle, "Biosensors and tools for surface functionalization from the macro- to the nanoscale: the way forward," Journal of Applied Physics, vol. 104, no. 11, pp. 111101-111116, 2008.

[2] A. M. Yates, S. J. Elvin, and D. E. Williamson, "The optimisation of a murine TNF- $\alpha$ ELISA and the application of the method to other murine cytokines," Journal of Immunoassay, vol. 20, no. 1-2, pp. 31-44, 1999.

[3] J. Renart, J. Reiser, and G. R. Stark, “Transfer of proteins from gels to diazobenzyloxymethyl-paper and detection with antisera: a method for studying antibody specificity and antigen structure," Proceedings of the National Academy of Sciences of the United States of America, vol. 76, no. 7, pp. 31163120, 1979.

[4] M. A. Lee, G. Brightwell, D. Leslie, H. Bird, and A. Hamilton, "Fluorescent detection techniques for real-time multiplex strand specific detection of Bacillus anthracis using rapid PCR," Journal of Applied Microbiology, vol. 87, no. 2, pp. 218223, 1999.

[5] L. B. Sagle, L. K. Ruvuna, J. A. Ruemmele et al., "Advances in localized surface plasmon resonance spectroscopy biosensing," Nanomedicine, vol. 6, no. 8, pp. 1447-1462, 2011.

[6] Z. Fu, S. Rogelj, and T. L. Kieft, "Rapid detection of Escherichia coli O157:H7 by immunomagnetic separation and real-time PCR," International Journal of Food Microbiology, vol. 99, no. 1, pp. 47-57, 2005. 
[7] J. E. Olsen, S. Aabo, W. Hill et al., "Probes and polymerase chain reaction for detection of food-borne bacterial pathogens," International Journal of Food Microbiology, vol. 28, no. 1, pp. 1-78, 1995.

[8] A. Vallée-Bélisle, F. Ricci, and K. W. Plaxco, "Engineering biosensors with extended, narrowed, or arbitrarily edited dynamic range," Journal of the American Chemical Society, vol. 134, no. 6, pp. 2876-2879, 2011.

[9] J. Zhao, X. Zhang, C. R. Yonzon, A. J. Haes, and R. P. Van Duyne, "Localized surface plasmon resonance biosensors," Nanomedicine, vol. 1, no. 2, pp. 219-228, 2006.

[10] A. J. Haes, W. P. Hall, L. Chang, W. L. Klein, and R. P. Van Duyne, "A localized surface plasmon resonance biosensor: first steps toward an assay for Alzheimer's disease," Nano Letters, vol. 4, no. 6, pp. 1029-1034, 2004.

[11] S. Link and M. A. El-Sayed, "Erratum: simulation of the optical absorption spectra of gold nanorods as a function of their aspect ratio and the effect of the medium dielectric constant (Journal of Physical Chemistry B (1999) 103B)," Journal of Physical Chemistry B, vol. 109, no. 20, pp. 1053110532, 2005.

[12] K. M. Mayer and J. H. Hafner, "Localized surface plasmon resonance sensors," Chemical Reviews, vol. 111, no. 6, pp. 3828-3857, 2011.

[13] E. Petryayeva and U. J. Krull, "Localized surface plasmon resonance: nanostructures, bioassays and biosensing-a review," Analytica Chimica Acta, vol. 706, no. 1, pp. 8-24, 2011.

[14] K. A. Willets and R. P. Van Duyne, "Localized surface plasmon resonance spectroscopy and sensing," Annual Review of Physical Chemistry, vol. 58, pp. 267-297, 2007.

[15] A. J. Haes, L. Chang, W. L. Klein, and R. P. Van Duyne, "Detection of a biomarker for Alzheimer's disease from synthetic and clinical samples using a nanoscale optical biosensor," Journal of the American Chemical Society, vol. 127, no. 7, pp. 2264-2271, 2005.

[16] S. Chen, M. Svedendahl, R. P. V. Duyne, and M. Käll, "Plasmon-enhanced colorimetric ELISA with single molecule sensitivity," Nano Letters, vol. 11, no. 4, pp. 1826-1830, 2011.

[17] T. Huang, P. D. Nallathamby, and X. H. N. Xu, "Photostable single-molecule nanoparticle optical biosensors for real-time sensing of single cytokine molecules and their binding reactions," Journal of the American Chemical Society, vol. 130, no. 50, pp. 17095-17105, 2008.

[18] S. Chen, M. Svedendahl, M. Käll, L. Gunnarsson, and A. Dmitriev, "Ultrahigh sensitivity made simple: nanoplasmonic label-free biosensing with an extremely low limit-of-detection for bacterial and cancer diagnostics," Nanotechnology, vol. 20, no. 43, Article ID 434015, 2009.

[19] Y. M. W. Zhou, H. Yang, Y. Ding, and X. Luo, "A labelfree biosensor based on silver nanoparticles array for clinical detection of serum p53 in head and neck squamous cell carcinoma," International Journal of Nanomedicine, vol. 6, no. 1, pp. 381-386, 2011.

[20] W. S. Hwang and S. J. Sim, "A Strategy for the ultrasensitive detection of cancer biomarkers based on the LSPR response of a single AuNP," Journal of Nanoscience and Nanotechnology, vol. 11, no. 7, pp. 5651-5656, 2011.

[21] J. B. González-Díaz, A. García-Martín, J. M. García-Martín et al., "Plasmonic $\mathrm{Au} / \mathrm{Co} / \mathrm{Au}$ nanosandwiches with enhanced magneto-optical activity," Small, vol. 4, no. 2, pp. 202-205, 2008.

[22] G. X. Du, T. Mori, M. Suzuki, S. Saito, H. Fukuda, and M. Takahashi, "Evidence of localized surface plasmon enhanced magneto-optical effect in nanodisk array," Applied Physics Letters, vol. 96, no. 8, article 081915, 3 pages, 2010.

[23] G. Mie, "Beitrge zur optik trüber medien, speziell kolloidaler metallösungen," Annalen Der Physik, vol. 330, no. 3, pp. 377445, 1908.

[24] E. Hutter and J. H. Fendler, "Exploitation of localized surface plasmon resonance," Advanced Materials, vol. 16, no. 19, pp. 1685-1706, 2004.

[25] J. Pérez-Juste, I. Pastoriza-Santos, L. M. Liz-Marzán, and P. Mulvaney, "Gold nanorods: synthesis, characterization and applications," Coordination Chemistry Reviews, vol. 249, no. 17-18, pp. 1870-1901, 2005.

[26] R. Gans, "Über die form ultramikroskopischer goldteilchen," Annalen Der Physik, vol. 342, no. 5, pp. 881-900, 1912.

[27] R. Gans, "Über die form ultramikroskopischer silberteilchen," Annalen Der Physik, vol. 352, no. 10, pp. 270-284, 1915.

[28] S. Link, M. B. Mohamed, and M. A. El-Sayed, "Simulation of the optical absorption spectra of gold nanorods as a function of their aspect ratio and the effect of the medium dielectric constant," Journal of Physical Chemistry B, vol. 103, no. 16, pp. 3073-3077, 1999.

[29] S. Eustis and M. A. El-Sayed, "Determination of the aspect ratio statistical distribution of gold nanorods in solution from a theoretical fit of the observed inhomogeneously broadened longitudinal plasmon resonance absorption spectrum," Journal of Applied Physics, vol. 100, no. 4, article 044324, 7 pages, 2006.

[30] T. R. Jensen, M. L. Duval, K. L. Kelly, A. A. Lazarides, G. C. Schatz, and R. P. Van Duyne, "Nanosphere lithography: effect of the external dielectric medium on the surface plasmon resonance spectrum of a periodic array of silver nanoparticles," Journal of Physical Chemistry B, vol. 103, no. 45, pp. 9846-9853, 1999.

[31] X. Lu, M. Rycenga, S. E. Skrabalak, B. Wiley, and Y. Xia, "Chemical synthesis of novel plasmonic nanoparticles," Annual Review of Physical Chemistry, vol. 60, pp. 167-192, 2009.

[32] L. M. Liz-Marzán, "Tailoring surface plasmons through the morphology and assembly of metal nanoparticles," Langmuir, vol. 22, no. 1, pp. 32-41, 2006.

[33] A. D. McFarland and R. P. Van Duyne, "Single silver nanoparticles as real-time optical sensors with zeptomole sensitivity," Nano Letters, vol. 3, no. 8, pp. 1057-1062, 2003.

[34] B. Sepúlveda, P. C. Angelomé, L. M. Lechuga, and L. M. LizMarzán, "LSPR-based nanobiosensors," Nano Today, vol. 4, no. 3, pp. 244-251, 2009.

[35] C. Xue, Z. Li, and C. A. Mirkin, "Large-scale assembly of single-crystal silver nanoprism monolayers," Small, vol. 1, no. 5, pp. 513-516, 2005.

[36] K. Fujiwara, H. Watarai, H. Itoh, E. Nakahama, and N. Ogawa, "Measurement of antibody binding to protein immobilized on gold nanoparticles by localized surface plasmon spectroscopy," Analytical and Bioanalytical Chemistry, vol. 386, no. 3, pp. 639-644, 2006.

[37] M. P. Kreuzer, R. Quidant, G. Badenes, and M. P. Marco, "Quantitative detection of doping substances by a localised surface plasmon sensor," Biosensors and Bioelectronics, vol. 21, no. 7, pp. 1345-1349, 2006.

[38] W. Rechberger, A. Hohenau, A. Leitner, J. R. Krenn, B. Lamprecht, and F. R. Aussenegg, "Optical properties of two interacting gold nanoparticles," Optics Communications, vol. 220, no. 1-3, pp. 137-141, 2003. 
[39] A. Boltasseva, "Plasmonic components fabrication via nanoimprint," Journal of Optics A, vol. 11, no. 11, article 114001, 2009.

[40] P. L. Stiles, J. A. Dieringer, N. C. Shah, and R. P. Van Duyne, "Surface-enhanced Raman spectroscopy," Annual Review of Analytical Chemistry, vol. 1, no. 1, pp. 601-626, 2008.

[41] A. Kosiorek, W. Kandulski, H. Glaczynska, and M. Giersig, "Fabrication of nanoscale rings, dots, and rods by combining shadow nanosphere lithography and annealed polystyrene nanosphere masks," Small, vol. 1, no. 4, pp. 439-444, 2005.

[42] H. M. Bok, K. L. Shuford, S. G. Kim, S. Kyukim, and S. Park, "Multiple surface plasmon modes for gold/silver alloy nanorods," Langmuir, vol. 25, no. 9, pp. 5266-5270, 2009.

[43] A. B. Dahlin, J. O. Tegenfeldt, and F. Höök, "Improving the instrumental resolution of sensors based on localized surface plasmon resonance," Analytical Chemistry, vol. 78, no. 13, pp. 4416-4423, 2006.

[44] P. Maruvada, W. Wang, P. D. Wagner, and S. Srivastava, "Biomarkers in molecular medicine: cancer detection and diagnosis," BioTechniques., supplement 9-15, 2005.

[45] H. Kitano, "Systems biology: a brief overview," Science, vol. 295, no. 5560, pp. 1662-1664, 2002.

[46] M. S. Wilson and W. Nie, "Multiplex measurement of seven tumor markers using an electrochemical protein chip," Analytical Chemistry, vol. 78, no. 18, pp. 6476-6483, 2006.

[47] S. Cesaro-Tadic, G. Dernick, D. Juncker et al., "Highsensitivity miniaturized immunoassays for tumor necrosis factor $\alpha$ using microfluidic systems," Lab on a Chip, vol. 4, no. 6, pp. 563-569, 2004.

[48] J. Yang, K. Eom, E. K. Lim et al., "In situ detection of live cancer cells by using bioprobes based on Au nanoparticles," Langmuir, vol. 24, no. 21, pp. 12112-12115, 2008.

[49] R. Polsky, R. Gill, L. Kaganovsky, and I. Willner, "Nucleic acid-functionalized Pt nanoparticles: catalytic labels for the amplified electrochemical detection of biomolecules," Analytical Chemistry, vol. 78, no. 7, pp. 2268-2271, 2006.

[50] A. J. Haes and R. P. Van Duyne, "A nanoscale optical biosensor: sensitivity and selectivity of an approach based on the localized surface plasmon resonance spectroscopy of triangular silver nanoparticles," Journal of the American Chemical Society, vol. 124, no. 35, pp. 10596-10604, 2002.

[51] N. Nath and A. Chilkoti, "A colorimetric gold nanoparticle sensor to interrogate biomolecular interactions in real time on a surface," Analytical Chemistry, vol. 74, no. 3, pp. 504-509, 2002.

[52] A. J. Haes, D. A. Stuart, S. Nie, and R. P. Van Duyne, "Using solution-phase nanoparticles, surface-confined nanoparticle arrays and single nanoparticles as biological sensing platforms," Journal of Fluorescence, vol. 14, no. 4, pp. 355-367, 2004.

[53] S. Zhu, F. Li, C. Du, and Y. Fu, "Novel bio-nanochip based on localized surface plasmon resonance spectroscopy of rhombic nanoparticles," Nanomedicine, vol. 3, no. 5, pp. 669-677, 2008.

[54] M. Kajiura, T. Nakanishi, H. Iida, H. Takada, and T. Osaka, "Biosensing by optical waveguide spectroscopy based on localized surface plasmon resonance of gold nanoparticles used as a probe or as a label," Journal of Colloid and Interface Science, vol. 335, no. 1, pp. 140-145, 2009.

[55] Y. Chuang, C. Y. Lee, S. H. Lu, S. C. Wang, L. K. Chau, and W. H. Hsieh, "Using ac-field-Lnduced electro-osmosis to accelerate biomolecular binding in fiber-optic sensing chips with microstructures," Analytical Chemistry, vol. 82, no. 3, pp. 1123-1127, 2010.
[56] M. Fan, M. Thompson, M. L. Andrade, and A. G. Brolo, "Silver nanoparticles on a plastic platform for localized surface plasmon resonance biosensing," Analytical Chemistry, vol. 82, no. 15, pp. 6350-6352, 2010.

[57] M. Wan, P. Luo, J. Jin, J. Xing, Z. Wang, and S. T. C. Wong, "Fabrication of localized surface plasmon resonance fiber probes using ionic self-assembled gold nanoparticles," Sensors, vol. 10, no. 7, pp. 6477-6487, 2010.

[58] Y. Lin, Y. Zou, Y. Mo et al., "E-Beam patterned gold nanodot arrays on optical fiber tips for localized surface plasmon resonance biochemical sensing," Sensors, vol. 10, no. 10, pp. 9397-9406, 2010.

[59] O. Vazquez-Mena, T. Sannomiya, L. G. Villanueva, J. Voros, and J. Brugger, "Metallic nanodot arrays by stencil lithography for plasmonic biosensing applications," ACS Nano, vol. 5, no. 2, pp. 844-853, 2011.

[60] G. Xiang, N. Zhang, and X. Zhou, "Localized surface plasmon resonance biosensing with large area of gold nanoholes fabricated by nanosphere lithography," Nanoscale Research Letters, vol. 5, no. 5, pp. 818-822, 2010.

[61] K. H. Chen, J. Hobley, Y. L. Foo, and X. Su, "Wide-field single metal nanoparticle spectroscopy for high throughput localized surface plasmon resonance sensing," Lab on a Chip, vol. 11, no. 11, pp. 1895-1901, 2011.

[62] T. Huang and X. H. N. Xu, "Multicolored nanometreresolution mapping of single protein-ligand binding complexes using far-field photostable optical nanoscopy (PHOTON)," Nanoscale, vol. 3, no. 9, pp. 3567-3572, 2011.

[63] K. Kaur and J. A. Forrest, "Influence of particle size on the binding activity of proteins adsorbed onto gold nanoparticles," Langmuir, vol. 28, no. 5, pp. 2736-2744, 2011.

[64] Y. Lin, Y. Zou, and R. G. Lindquist, "A reflection-based localized surface plasmon resonance fiber-optic probe for biochemical sensing," Biomedical Optics Express, vol. 2, no. 3, pp. 478-484, 2011.

[65] T. Endo, K. Kerman, N. Nagatani et al., "Multiple label-free detection of antigen-antibody reaction using localized surface plasmon resonance-based core-shell structured nanoparticle layer nanochip," Analytical Chemistry, vol. 78, no. 18, pp. 6465-6475, 2006

[66] R. Tanaka, T. Yuhi, N. Nagatani et al., "A novel enhancement assay for immunochromatographic test strips using gold nanoparticles," Analytical and Bioanalytical Chemistry, vol. 385, no. 8, pp. 1414-1420, 2006.

[67] B. Y. Hsieh, Y. F. Chang, M. Y. Ng et al., "Localized surface plasmon coupled fluorescence fiber-optic biosensor with gold nanoparticles," Analytical Chemistry, vol. 79, no. 9, pp. 34873493, 2007.

[68] T. A. Bendikov, A. Rabinkov, T. Karakouz, A. Vaskevich, and I. Rubinstein, "Biological sensing and interface design in gold island film based localized plasmon transducers," Analytical Chemistry, vol. 80, no. 19, pp. 7487-7498, 2008.

[69] T. Endo, S. Yamamura, K. Kerman, and E. Tamiya, "Labelfree cell-based assay using localized surface plasmon resonance biosensor," Analytica Chimica Acta, vol. 614, no. 2, pp. 182189, 2008.

[70] K. M. Mayer, S. Lee, H. Liao et al., "A label-free immunoassay based upon localized surface plasmon resonance of gold nanorods," ACS Nano, vol. 2, no. 4, pp. 687-692, 2008.

[71] J. N. Anker, W. P. Hall, M. P. Lambert et al., "Detection and identification of bioanalytes with high resolution LSPR spectroscopy and MALDI mass spectrometry," Journal of Physical Chemistry C, vol. 113, no. 15, pp. 5891-5894, 2009. 
[72] L. Au, Q. Zhang, C. M. Cobley et al., "Quantifying the cellular uptake of antibody-conjugated au nanocages by twophoton microscopy and inductively coupled plasma mass spectrometry," ACS Nano, vol. 4, no. 1, pp. 35-42, 2010.

[73] C. Huang, K. Bonroy, G. Reekmans et al., "Localized surface plasmon resonance biosensor integrated with microfluidic chip," Biomedical Microdevices, vol. 11, no. 4, pp. 893-901, 2009.

[74] S. Zheng, D. K. Kim, T. J. Park, S. J. Lee, and S. Y. Lee, "Labelfree optical diagnosis of hepatitis B virus with genetically engineered fusion proteins," Talanta, vol. 82, no. 2, pp. 803$809,2010$.

[75] H. Huang, S. Huang, S. Yuan et al., "High-sensitivity biosensors fabricated by tailoring the localized surface plasmon resonance property of core-shell gold nanorods," Analytica Chimica Acta, vol. 683, no. 2, pp. 242-247, 2011.

[76] D. K. Kim, T. J. Park, E. Tamiya et al., "Label-free detection of leptin antibody-antigen interaction by using LSPR-based optical biosensor," Journal of Nanoscience and Nanotechnology, vol. 11, no. 5, pp. 4188-4193, 2011.

[77] S. W. Lee, K. S. Lee, J. Ahn, J. J. Lee, M. G. Kim, and Y. B. Shin, "Highly sensitive biosensing using arrays of plasmonic $\mathrm{Au}$ nanodisks realized by nanoimprint lithography," ACS Nano, vol. 5, no. 2, pp. 897-904, 2011.

[78] P. L. Truong, C. Cao, S. Park, M. Kim, and S. J. Sim, "A new method for non-labeling attomolar detection of diseases based on an individual gold nanorod immunosensor," Lab on a Chip, vol. 11, no. 15, pp. 2591-2597, 2011.

[79] J. Yamamichi, T. Ojima, K. Yurugi et al., "Single-step, labelfree quantification of antibody in human serum for clinical applications based on localized surface plasmon resonance," Nanomedicine, vol. 7, no. 6, pp. 889-895, 2011.

[80] S. H. Yeom, O. G. Kim, B. H. Kang et al., "Highly sensitive nano-porous lattice biosensor based on localized surface plasmon resonance and interference," Optics Express, vol. 19, no. 23, pp. 22882-22891, 2011.

[81] L. Guo and D. H. Kim, "LSPR biomolecular assay with high sensitivity induced by aptamer-antigen-antibody sandwich complex," Biosensors and Bioelectronics, vol. 31, no. 1, pp. 567570, 2012.

[82] T. J. Park, S. J. Lee, D. K. Kim et al., "Development of label-free optical diagnosis for sensitive detection of influenza virus with genetically engineered fusion protein," Talanta, vol. 89, no. 0 , pp. 246-252, 2012. 

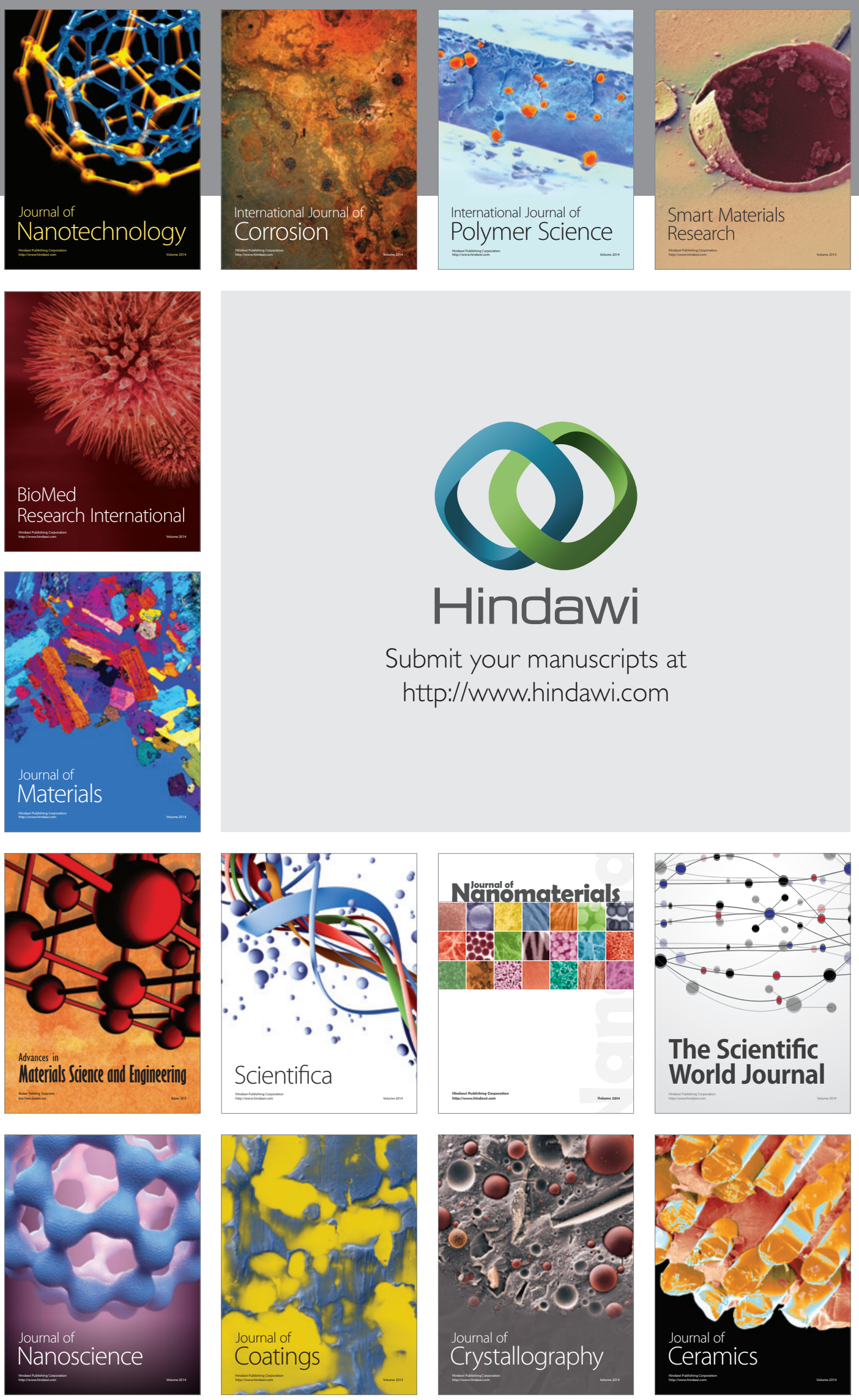

The Scientific World Journal

Submit your manuscripts at

http://www.hindawi.com

\section{World Journal}

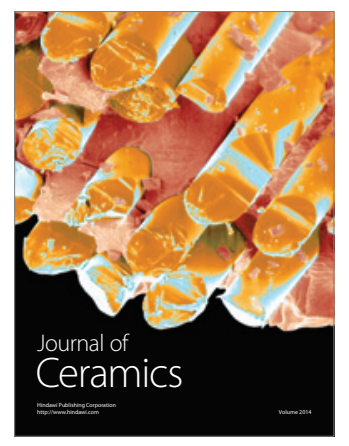

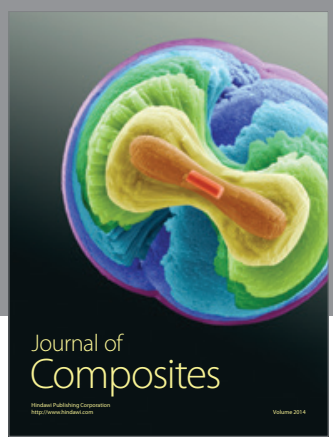
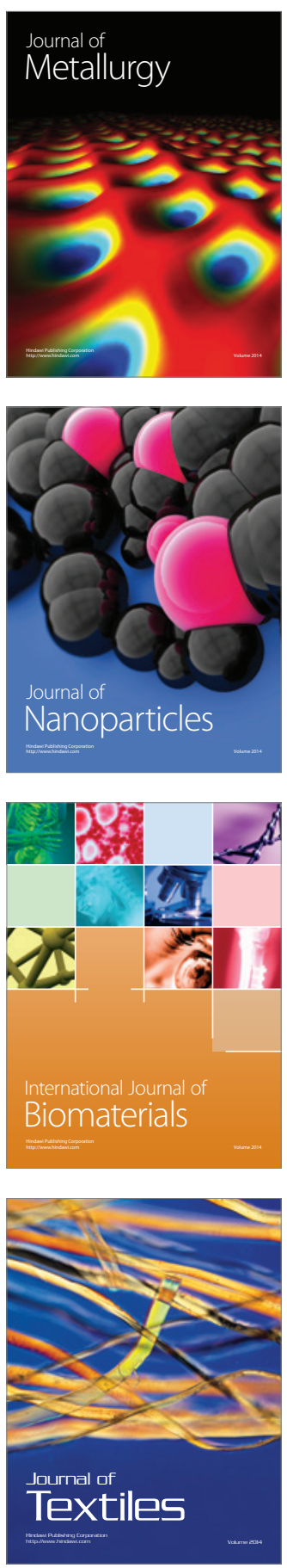